\title{
Comparison of skill in novice nurses before and after venipuncture simulation practice
}

\author{
Chieko Fujii \\ Faculty of Nursing and Medical Care, Keio University, Kanagawa, Japan. \\ Correspondence: Chieko Fujii. Address: 4411, Endo, Fujisawa-City, Kanagawa, 2520883, Japan. Email: \\ chieko@sfc.keio.ac.jp
}

Received: December 15, 2013

Accepted: January 25, $2014 \quad$ Online Published: February 23, 2014

DOI : $10.5430 /$ jnep.v4n5p16

URL: http://dx.doi.org/10.5430/jnep.v4n5p16

\section{Abstract}

Background: To carry out a successful venipuncture it is necessary to identify a specific point on a vein and maintain stability of the needle tip.

Methods: This study compared the motion of straight and butterfly needles during venipunctures carried out by novice nurses before and after educational intervention. The nurses were videotaped whilst performing venipunctures on a simulated model arm.

Results: The findings of the study showed that butterfly needles were inserted at a greater angle than straight needles. The use of straight needles involved a shorter venipuncture time, with the puncture points being closer together following educational intervention. However, the distance butterfly needles moved during venipuncture was greater after intervention. It appeared that picking-up the wing of the needle caused instability, leading the nurses to place their hand on the model and to have difficulties performing the needle stab. After educational intervention the nurses were able to advance the needle into a blood vessel.

Conclusions: Skin at the puncture point extends and may enter the inside of a vein. It is therefore important to maintain an appropriate puncture point and angle without shaking or rotating the needle. It is also necessary to practice to achieve stable movements of the needle and appropriate movements of the hand.

\section{Key words}

Butterfly needle, Straight needle, Simulation, Nursing practice, Novice nurse

\section{Introduction}

Appropriate attention to system factors that contribute to errors and design of intelligent systems may help control and eliminate many errors in health care ${ }^{[1]}$. The majority of errors during venous blood testing result from human mistakes that occur before the sample reaches the laboratory. Given the large number of venous blood samples collected, preanalytical errors have the potential to cause an unacceptably high number of adverse effects on patient care ${ }^{[2]}$. Performing venipuncture and starting intravenous infusions are among the most challenging clinical skills nurses are required to master. Nurses also review laboratory records, which often identify procedures, conditions, and even individual clinicians who were associated with blood samples that could not be processed or testing results that were inaccurate ${ }^{[3]}$. 
Complications resulting from venipuncture include nerve damage, hematomas, and neuropathic pain. The occupational transmission of blood-borne viruses through a needle-stick injury (NSI) has been recognized widely over the past 20 years ${ }^{[4]}$. NSIs are a significant potential risk with the use of sharp devices. The incidence of NSIs and the costs associated with their management can be reduced through the adoption of safer work practices ${ }^{[5]}$. Although there is considerable information on the incidence and causes of needle-stick and sharps injuries in trained nurses, there is a lack of similar information in the student nursing population ${ }^{[6]}$. Only a few nursing schools offer hands-on learning, while hospitals typically provide only limited opportunities for supervised practice ${ }^{[7]}$. Nursing school students are particularly susceptible to occupational needle-stick injuries due to their limited clinical experience ${ }^{[8]}$. Whilst performing their clinical assignments in hospitals, medical students are also at risk of needle-stick injury and the possibility of developing infections from blood-borne pathogens such as the human immunodeficiency and hepatitis B and C viruses ${ }^{[9]}$.

Occupational exposure to blood-borne pathogens remains an important and largely preventable issue in hospital practice. For the first attempt at venipuncture it is necessary to consider what equipment should be used and how the procedure should be carried out.

The most common devices used for venipuncture are syringes or vacuum-tube holders, both of which can be connected to straight or butterfly needles. Basic training for venipuncture in Japan has focused mainly on the syringe method. In this method blood flows into the needle hub the instant the needle enters the vein, whereas when a vacuum tube is used it cannot be ascertained whether the needle has entered the vein until blood enters the tube. The butterfly devices design allows for ease of multiple tube collection, but the short needle length limits their utility to surface veins ${ }^{[10]}$. Collection systems where blood is drawn through butterfly needles may result in significantly more hemolysis than blood obtained by venipuncture using conventional needles ${ }^{[1]}$. The use of butterfly needles is recommended when puncturing sites that have a high risk of causing nerve injury ${ }^{[12]}$. However, the education methods for using butterfly needle have not been fully established. A safety strategy is therefore needed, and in order to develop appropriate venipuncture procedures, nurses should take advantage of these devices ${ }^{[13]}$.

It is necessary to develop programs for the initial education of nurses. Clinical simulation is now having a significant impact on health-care education across professional boundaries in both the undergraduate and postgraduate arenas. However, the quantity and quality of research on education involving simulation remains limited ${ }^{[14]}$. Simulation training with forced feedback has been shown to significantly improve measurement of cricoid pressure in the clinical setting. It has also been proposed that simulation training should be used more frequently to train and maintain resuscitation skills ${ }^{[15]}$. It is therefore important that a link is made between this literature and venipuncture simulation.

Problems commonly observed during venipuncture include needle movements during puncture and insertion, involving rotation and insertion in a wave-like trajectory. Appropriate training, including recommendations to maintain stability of the needle tip, is therefore important to ensure safety during venipuncture ${ }^{[16]}$. It is necessary to define a point on the vein with the needle and focus carefully on needling this point. The aim of this study was to compare the movements of straight and butterfly needles during venipuncture carried out by nurses before and after educational intervention.

\section{Methods}

\subsection{Subjects}

The study was carried out in a hospital in Japan in August 2012. Nine novice nurses with three months of post-registration work experience participated in the first investigation. The participants performed five venipunctures using a $20-\mathrm{ml}$ syringe and either straight or butterfly needles. The participants were given the following instructions after the first demonstration; "Consciously insert the needle towards the same point, and be aware of the need to reduce turning the 
wrist." Each nurse practiced these instructions by themselves. Ten days later, the same demonstration was carried out and self-reporting including data on age were recorded. Seven of the 9 participants performed these venipunctures.

\subsection{Venipuncture device and simulator model}

The injection needles used in the study were a 22-gauge needle $(0.70 \mathrm{~mm} \times 32 \mathrm{~mm})$ and a butterfly needle $(0.70 \mathrm{~mm} \times 19$ $\mathrm{mm}$ ) manufactured by NIPRO Corporation (Osaka, Japan).

A strap-on venipuncture trainer model (Kyotokagaku; M87 11326-000, Kyoto, Japan) was used to simulate the venipuncture procedure, and consisted of a main body containing a tube covered by simulated skin. The nurses confirmed that this tube (i.e., vein) could be palpated through the skin surface. This investigation was limited to the measurement of only the needle during venipuncture. Therefore, the needle holes made in the centre of the tube enabled the nurse to observe air being drawn into the syringe.

\subsection{Data processing procedure}

The investigations were carried out with the cooperation of the hospital staff, with the assurance that the results were not included in the evaluation of the nurse's duties. We positioned a video camera $50 \mathrm{~cm}$ from the trainer model. The video film photographed only their hands without photographing the face. The researcher recorded the venipunctures using a video camera.

Venipuncture techniques were categorized based on individual frames in video images, with the $\mathrm{X}$ and $\mathrm{Y}$ coordinates graphed using WINanalyze software (Mikromak GmbH, Germany). This software tracks objects without the necessity for markers. Time was eliminated by dividing the number of frames by 59.94 .

Data were transferred into AVI files from a mini High-Definition Digital Video Cassette. The SSD-correlation tracking algorithm was activated during acquisition to adapt for color differences in the images, with the image sequence function being used to display the connections and objects in all the frames. The $\mathrm{X}$ and $\mathrm{Y}$ coordinates were displayed on the vertical axis and time on the horizontal axis. Selecting objects in all the frames allowed needle movement during multiple punctures to be displayed simultaneously. Each puncture could also be displayed individually. Figure 1 shows the points used for tracking.

Figure 1. The points used for tracking

Point A: Contact between the needle and the model.

Point B: Needle movement measured at the welded part of the hub.

Point C: Metatarsophalangeal refers to the toe joints (MP joint) of the first finger.

Point D: MP joint of the second finger.

Points $\mathrm{E}$ and F: Confirmation of the $5 \mathrm{~cm}$ width.

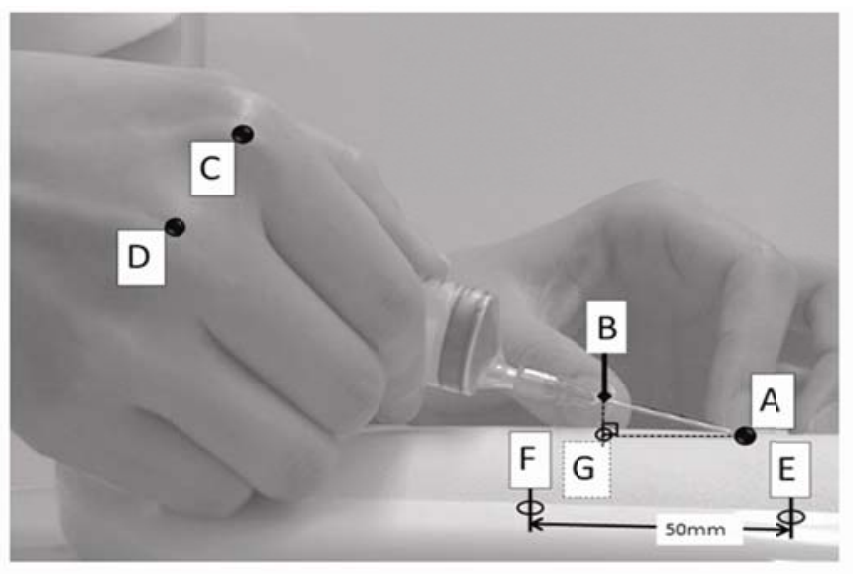

\subsection{Observation parameters}

1) Angle BAG ( $G$ is Point A of the needle relative to the parallel surface, with angle $A G B=90^{\circ}$ ). Angles $C A G$ and $D A G$ were measured at times (a) and (b), respectively and were calculated from the $X-Y$ coordinates at times (a) and (b) as: atan2 $\left(\mathrm{Y}_{\mathrm{b}}-\mathrm{Y}_{\mathrm{a}}, \mathrm{X}_{\mathrm{b}}-\mathrm{X}_{\mathrm{a}}\right)$. Angle BAG represents the puncture angle. 
2) Time of needle movement: The number of frames from time (b) to time (a) were counted and the total reduced by 1 . The number of seconds was calculated by dividing the result by 59.94 .

3) The distance travelled by the needle was calculated as: $\sqrt{ }\left(\left(X_{2}-X_{1}\right)^{2}+\left(Y_{2}-Y_{1}\right)^{2}\right)$, using the $X-Y$ coordinates for times

(b) and (a), respectively. The amount of motion occurring from one frame to the next was calculated.

4) The distances between puncture points during multiple punctures were also measured.

\subsection{Data analysis}

Data were analyzed using SPSS software (SPSS 19.0 for Windows; IBM, Japan). Paired $t$-tests were used to examine the data collected before- and after educational intervention. $P<.05$ were considered significant.

\subsection{Ethical approval}

Ethical approval was obtained from the ethics committee of the Graduate School of Health Management, Keio University. The participants were provided with an explanation of the study objectives and were informed they would not be penalized for withdrawing from the study.

\section{Results}

\subsection{Differences in venipuncture technique before and after intervention}

The seven participants had a mean age ( \pm standard deviation) of $22.9 \pm 3.3$ years. Table 1 shows the differences in observation parameters before and after educational intervention. The angle of the straight needle, which represented puncture angle, was $19.3 \pm 6.3^{\circ}$ before intervention and $19.5 \pm 6.5^{\circ}$ after intervention, while the corresponding angles for the butterfly needle were $26.3 \pm 7.3^{\circ}$ and $26.7 \pm 7.9^{\circ}$. The time of needle movement for the straight needle was $4.3 \pm 1.6 \mathrm{~s}$ before intervention and $3.7 \pm 1.7 \mathrm{~s}$ after intervention $(P=.018)$. The mean distance between puncture points during multiple punctures using a straight needle was $4.6 \pm 3.7 \mathrm{~mm}$ before intervention and $2.4 \pm 1.7 \mathrm{~mm}$ after intervention $(P=$ $.004)$.

Table 1. Differences in before and after intervention

\begin{tabular}{|c|c|c|c|c|c|c|c|c|}
\hline & \multicolumn{4}{|c|}{ Straight needle } & \multicolumn{4}{|c|}{ Butterfly needle } \\
\hline & $\mathbf{n}$ & Before & After & $P^{* 1}$ & $\mathbf{n}$ & Before & After & $P^{* 1}$ \\
\hline \multicolumn{9}{|l|}{ Angle(degree) } \\
\hline Contact between the needle and model & 26 & $19.3(6.3)$ & $19.5(6.5)$ & .812 & 26 & $26.3(7.3)$ & $26.7(7.9)$ & .786 \\
\hline MP joint of the first fingers & 26 & $30.1(9.1)$ & $29.2(5.5)$ & .619 & 26 & $57.1(7.7)$ & $53.1(10.4)$ & .346 \\
\hline MP joint of second fingers & 26 & $15.1(11.0)$ & $13.4(7.9)$ & .330 & 26 & $41.0(7.2)$ & $36.4(8.8)$ & .171 \\
\hline \multicolumn{9}{|l|}{ Time(second) } \\
\hline Time of the needle movement & 26 & $4.3(1.6)$ & $3.7(1.7)$ & .018 & 26 & $2.6(0.7)$ & $2.5(1.0)$ & .604 \\
\hline \multicolumn{9}{|l|}{ Movement distance(mm) } \\
\hline Needle movement & 26 & $20.2(7.3)$ & $19.2(6.1)$ & .574 & 25 & $11.7(2.2)$ & $14.0(3.9)$ & .006 \\
\hline Needle movement; First one second & 26 & $6.2(2.6)$ & $6.7(3.9)$ & .366 & 25 & $5.2(2.0)$ & $6.7(2.4)$ & .001 \\
\hline Puncture points during multiple puncture & 26 & $4.6(3.7)$ & $2.4(1.7)$ & .004 & 22 & $2.9(2.1)$ & $4.6(4.4)$ & .149 \\
\hline
\end{tabular}

${ }^{*}$ : Paired- t test; Mean(Standard Deviation)

Movement of the butterfly needle was $11.7 \pm 2.2 \mathrm{~mm}$ before intervention and $14.0 \pm 3.9 \mathrm{~mm}$ after intervention $(P=.006)$, while first needle movement was $5.2 \pm 2.0 \mathrm{~mm}$ before intervention and $6.7 \pm 2.4 \mathrm{~mm}$ after intervention $(P=.001)$. Before 
intervention, the nurses only placed the needle on the model, whereas after practice they advanced the needle towards the blood vessel.

\subsection{Case study}

Case 1 shows stable movement of the needle after training and carrying out the practice exercises shown in Figure 2. Figure 3 shows time-related changes during venipuncture in another case. In the $1^{\text {st }}$ to the $5^{\text {th }}$ attempts, needle movement was limited because of the forward position of the finger under the syringe. By turning the wrist and pointing the fingers upwards, the nurse was able to move the needle into the simulated arm. A commonly observed outcome of this technique involved the nurse lifting the needle above the top of the skin and therefore in order to penetrate the skin, power needed to be exerted to the middle of the needle, causing it to bend downwards. When power was then exerted through the needle hub to advance the needle into the blood vessel this caused the needle to bend upwards. On the $6^{\text {th }}$ attempt the nurse changed the position of her hand to avoid these problems.

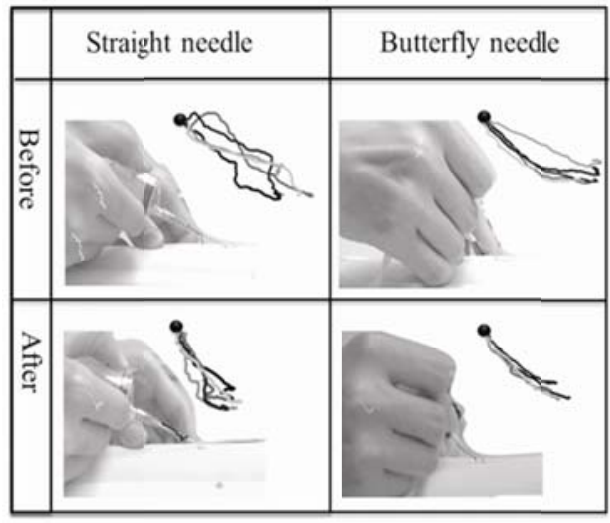

Figure 2. Case study. Case 1: Movement was stabilized by practice.

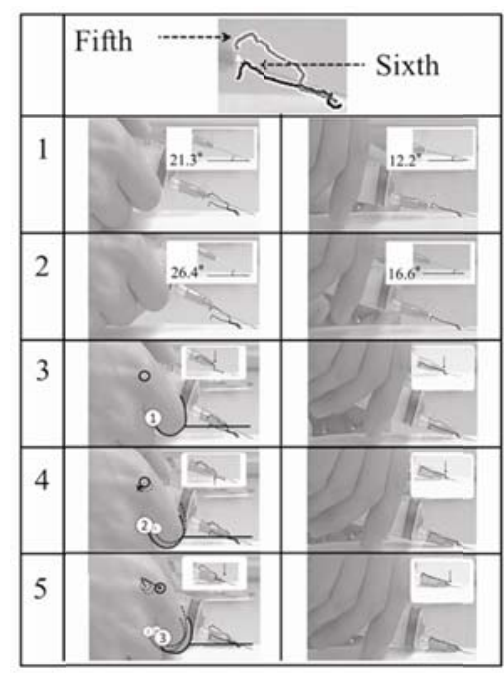

Figure 3. Time-related changes during venipuncture Case 2: A different technique was added at the $6^{\text {th }}$ attempt, with a change in the method used to hold the needle.

Images 1 and 2 show a raised puncture angle for insertion of the needle.

Images 3,4 , and 5 of the 5 th attempt showed the hand being turned.

\section{Discussion}

As novice nurses make the transition into clinical practice, their critical thinking and understanding have been described as acontextual, in which they use rule-governed thinking to solve practice problems ${ }^{[17]}$. Precise skill is necessary to perform blood collection that is safe for both patients and nurses. However, nurses in Japan often lack practice before attempting venipuncture for the first time, at both the pre and post-licensure times. It is therefore important that we consider educational steps for nursing students and novice nurses.

Training on a simulator resulted in the nurses being able to move the needle in a straight direction in a shorter time. This suggested that unnecessary rolling of the needle had decreased, and resulted in the points of insertion being closer together. Before intervention, the characteristic movement was to draw the needle in an arc, with power being applied in the center of the arc. Training emphasized the importance of consciously needling the same point, with the aim of centralizing power 
to the tip of the needle. The nurses were therefore required to needle the identified point by focusing on the needle tip. This strategy proved effective as a first learning experience.

It is necessary for nurses to learn venipuncture skills in order to minimize risks to the patient. The first attempt at venipuncture on a patient should be easy, with prominent veins being visible on the surface. Puncture using a butterfly needle is performed whilst holding both wings with the first and second fingers. The needle and wings are therefore integrated and venipuncture can be performed easily on surface veins. As the butterfly consists of a winged needle and plastic tubing, the vacuum-tube holder or syringe are separated from the needle by tubing. However, our study showed it was easier to puncture the skin using a straight needle than with a butterfly needle, and that the butterfly needle was inserted at a greater angle than the straight needle. The MP joint of the first and second fingers also showed a higher angle with the butterfly needle.

In order to prevent nerve injuries during venipuncture it is first necessary to retract the skin and stabilize the vein, before inserting the needle at a 5 to $15^{\circ}$ angle. The angle is then lowered to thread the catheter into the vein ${ }^{[18]}$. However, in our study it proved difficult to penetrate the skin of the model using this lower angle. When the needle angle was raised this resulted in the nurses often placing their hand on the arm. It is dangerous for the needle to be inserted too deeply as it is possible to cause nerve damage and also not result in entry into the vein. The area of the fifth metacarpal bone was attached to the simulator model so that the average angle of puncture using a butterfly needle was $26.3^{\circ}$. The angle of each point lowered after educational intervention. Prior to intervention, the nurses placed their fifth metacarpal bone on the model, and had limited ability to move the needle forward in small steps.

After educational intervention there was an increase in the distance the needle moved. We also showed movement was limited by using some fingers for stability. In order to lower the angle of the needle the nurses were required to turn their hand upwards, which resulted in the entire needle turning and being poorly positioned within the blood vessel. Practice to ensure needle stability was necessary when a finger was not placed on the skin to maintain stability.

Frequent venipunctures usually cause pain, skin damage, and superficial bleeding in patients ${ }^{[19]}$. It is necessary for nurses to become used to handling needles. The benefit of using simulation exercises as an adjunct to didactic teaching was described in a report on hospital nurse educators who used simulation methodology to educate nurses on the complexity of safe administration of medication and the importance of critical thinking in daily practice ${ }^{[20]}$. Although simulated clinical experience is now being used increasingly in nursing education, vital evidence on knowledge acquisition associated with simulated clinical experience does not exist ${ }^{[21]}$. Simulation programs can play an important role in promoting patient safety ${ }^{[22]}$. For example, if a nurse requires correction of a technique they are able to repeat the procedure without assistance from another person.

Initially, it is necessary for a student or novice nurse to become familiar with the needle puncture itself and this level of skill can be attained on the simulator before carrying out a venipuncture on a patient. The nurses can also use a finger for stability as this prevents adverse movements. For appropriate insertion of the needle into a vein it is also necessary to consider whether to move the hand.

\section{Conclusion}

It is necessary to use the simulator effectively in order to minimize risks to the patient. Although it is generally considered that it is easy to use a butterfly needle, we showed that these needles are inserted at a greater angle than straight needles. Because of this increase in needle angle nurses often placing their hand on the patient's arm.

Although the practice exercise was for only 10 days, movement of the needle became fluent during this time. A point to be noted before exercise involved focusing towards the same point of insertion and not applying power to the middle of the 
needle. In other words, this involved not rolling the needle and concentrating power to the needle tip, resulting in minimal movement of the needle. Initially, it was necessary for a student or novice nurse to become familiar with the needle puncture itself.

The study had several limitations as only a small number of nurses participated in the investigations, and only needle movement during the puncture was evaluated. In future studies it would be desirable to collect artificial blood from the veins using simulation techniques.

\section{References}

[1] Kalra, J. Medical errors: impact on clinical laboratories and other critical areas. Clin Biochem. 2004; 37(12): 1052-1062. http://dx.doi.org/10.1016/j.clinbiochem.2004.08.009

[2] Wallin, O., Söderberg, J., Van Guelpen, B., Stenlund, H., Grankvist, K., Brulin, C. Blood sample collection and patient identification demand improvement: a questionnaire study of preanalytical practices in hospital wards and laboratories. Scand J Caring Sci. 2010; 24(3): 581-591. http://dx.doi.org/10.1111/j.1471-6712.2009.00753.x

[3] Bowe, G., Leigh, A. 2011. Lower volume peripheral blood sample collection: Key considerations for nurses - Roundtable Discussion. JAVA. 2011; 16(3): 150-154. http://dx.doi.org/10.2309/java.16-3-4

[4] Slater, K., Whitby, M., McLaws, M.L. Prevention of needlestick injuries: the need for strategic marketing to address health care worker misperceptions. Am J Infect Control. 2007; 35(8): 560-562. http://dx.doi.org/10.1016/j.ajic.2006.12.007

[5] Hanmore, E., Maclaine, G., Garin, F., Alonso, A., Leroy, N., Ruff, L. Economic benefits of safety-engineered sharp devices in Belgium a budget impact model. BMC Health Serv Res. 2013; 13:489. http://dx.doi.org/10.1186/1472-6963-13-489

[6] Hambridge, K. 2011. Needlestick and sharps injuries in the nursing student population. Nurs Stand. 2011; 25(27): $38-45$. http://dx.doi.org/10.7748/ns2011.03.25.27.38.c8389

[7] Millam, D. A. Starting I.V.s: how to develop your venipuncture expertise. Nursing. 1992; 22(9): 33-48. http://dx.doi.org/10.1097/00152193-199209000-00010

[8] Yao, W.X, Yang, B., Yao, C., Bai, P.S., Qian, Y.R., Huang, C.H., Liu, M. Needlestick injuries among nursing students in China. Nurse Educ Today. 2010; 30(5): 435-437. http://dx.doi.org/10.1016/j.nedt.2009.09.018

[9] Mohamad, Y.N., Ismail, N.H. Study on incidence of needle stick injury and factors associated with this problem among medical students. J Occup Health. 2003; 45(3): 172-178. http://dx.doi.org/10.1539/joh.45.172

[10] Bowen, R,A., Hortin, G,L., Csako, G., Otañez, O,H., Remaley, A,T. Impact of blood collection devices on clinical chemistry assays. Clin Biochem. 2010; 43(1-2): 4-25. http://dx.doi.org/10.1016/j.clinbiochem.2009.10.001

[11] Loeffen, R., Kleinegris, M.C., Loubele, S.T., Pluijmen, P.H., Fens, D., van Oerle, R., ten Cate, H., Spronk, H.M,. Preanalytic variables of thrombin generation: towards a standard procedure and validation of the method. J Thromb Haemost. 2012; 10(12): $2544-2554$. http://dx.doi.org/10.1111/jth.12012

[12] Ohnishi, H., Watanabe, M., Watanabe, T. Butterfly needles reduce the incidence of nerve injury during phlebotomy. Arch Pathol Lab Med. 2012; 136(4): 352. http://dx.doi.org/10.5858/arpa.2011-0431-LE

[13] Ford, J., Phillips, P. An evaluation of sharp safety blood evacuation devices. Nurs Stand. 2007; 25(43): 41-47. http://dx.doi.org/10.7748/ns2011.06.25.43.41.c8595

[14] Bradley, P. The history of simulation in medical education and possible future directions. Med Educ. 2006; 40(3): $254-262$. http://dx.doi.org/10.1111/j.1365-2929.2006.02394.x

[15] Domuracki, K.J., Moule, C.J., Owen, H., Kostandoff, G., Plummer, J.L. Learning on a simulator does transfer to clinical practice. Resuscitation. 2009; 80(3): 346-349. http://dx.doi.org/10.1016/j.resuscitation.2008.10.036

[16] Fujii, C., Ishii, H., Takanishi, A. Safe venipuncture techniques using a vacuum tube system. Int Nurs Pract. 2013; 19(Suppl 3): 11-19.

[17] Forneris, S.G., Peden-McAlpine, C. Evaluation of a reflective learning intervention to improve critical thinking in novice nurses. J Adv Nurs. 2007; 57(4): 410-421. http://dx.doi.org/10.1111/j.1365-2648.2007.04120.x

[18] Masoorli, S., Angeles, T., Barbone, M. Danger points. How to prevent nerve injuries from venipuncture. Nursing. 1998; 28(9): 34-39.

[19] Cicolini, G., Simonetti, V., Di Nicola, M., Palma, E. Comparison of blood samples values by direct venipuncture and Central Venous Catheters with 5 or $10 \mathrm{ml}$ wasted blood. J Clin Nurs. 2012; 21(1-2): 281-284. http://dx.doi.org/10.1111/j.1365-2702.2011.03988.x

[20] Paparella, S.F., Mariani, B. A., Layton, K., Carpenter, A.M. Patient safety simulation: learning about safety never seemed more fun. J Nursws Staff Dev. 2004; 20(6): 247-252. http://dx.doi.org/10.1097/00124645-200411000-00001

[21] Schlairet, M.C., Pollock, J.W. Equivalence testing of traditional and simulated clinical experiences: undergraduate nursing students' knowledge acquisition. J Nurs Educ. 2010; 49(1): 43-47. http://dx.doi.org/10.3928/01484834-20090918-08

[22] Long, R.E. Using simulation to teach resuscitation: an important patient safety tool. Crit Care Nurs Clin North Am. 2005 ; $17(1)$ : 1-8. http://dx.doi.org/10.1016/j.ccell.2004.09.001 